\title{
Inverse opal nanocrystal superlattice films
}

\author{
Aaron E. Saunders ${ }^{\dagger}$, Parag S. Shah ${ }^{\dagger}$, Michael B. Sigman, Jr. ${ }^{\dagger}$, Tobias Hanrath ${ }^{\dagger}$, \\ Ha Soo Hwang ${ }^{\ddagger}$, Kwon T. Lim, Keith P. Johnston ${ }^{\dagger}$, Brian A. Korgel ${ }^{\dagger, *}$
}

\footnotetext{
${ }^{\dagger}$ Department of Chemical Engineering and Texas Materials Institute, Center for Nano- and Molecular Science and Technology, The University of Texas, Austin, TX 78712, USA.

* Department of Imaging Information Science \& Engineering, Pukyong National University, Pusan, Korea

* Corresponding author: E-mail: korgel@che.utexas.edu. (T) 512-471-5633. (F) 512-471-7060.
}

\section{Supporting Information: Experimental Techniques and Characterization Methods}

\section{Gold Nanocrystal Synthesis}

Gold nanocrystals passivated with fluorinated ligands were prepared using previously described methods. ${ }^{1,2}$ Initially, $18 \mathrm{~mL}$ of aqueous $(0.027 \mathrm{M})$ hydrogen tetrachloroaurate (III) trihydrate was combined with $12 \mathrm{~mL}$ of a $0.207 \mathrm{M}$ chloroform solution containing tetraoctylammonium bromide, a phase transfer catalyst. Stirring the solution for one half hour completely transferred the gold ions from the aqueous to the organic layer, after which the aqueous phase was removed. Under vigorous stirring, the gold salt was reduced using $15 \mathrm{~mL}$ of an aqueous $(0.441 \mathrm{M})$ solution of sodium borohydride. The solution was stirred overnight, resulting in polydisperse gold nanocrystals with diameters between 2 and $6 \mathrm{~nm}$, protected by the phase transfer catalyst. The aqueous phase was removed. Addition of a fluorinated thiol (either perfluoropolyether thiol (PFPE-SH) or fluorooctyl methacrylate thiol (FOMA-SH)) causes replacement of the weakly binding amine on the gold surface with the more strongly binding thiol. The fluorinated-thiol passivated nanocrystals are not soluble in chloroform and precipitate from solution; the nanocrystals are easily separated from the reaction mixture via centrifugation and can be redispersed into 1,1,2-trichlorotrifluoroethane (Freon 113).

Size-selection of the polydisperse nanocrystals was accomplished through the addition of a small amount of a miscible antisolvent (hexane) to the Freon dispersion, resulting in precipitation of the largest nanocrystals which could be isolated by centrifugation. Repeating 
this procedure produced fractions of monodisperse nanocrystals with decreasing diameter. A TEM micrograph of the nanocrystals used in the study is shown in Figure S1.

The concentration of the dispersions was varied between 1.3 and $9.1 \mathrm{mg} / \mathrm{mL}$ by diluting a concentrated dispersion with pure Freon. The concentration of each dispersion was determined by comparing the intensity of the plasmon peak of the gold nanocrystals (measured using a Cary $500 \mathrm{UV}-\mathrm{Vis}-\mathrm{NIR}$ spectrophotometer) with a calibration curve.

\section{Nanocrystal film formation}

The inverse-opal structured nanocrystal films were formed by allowing a droplet of dispersion to evaporate under a stagnant atmosphere at controlled temperature and relative humidity. All experiments were carried out at $24^{\circ} \mathrm{C}$ with relative humidity values between $30 \%$ and $95 \%$.

Humidity was controlled by evaporating water in a closed system via mild heating until the desired conditions had been reached; the system was allowed to sit for at least one hour to reach equilibrium conditions.

For analysis by TEM, $3 \mu \mathrm{L}$ of dispersion was deposited onto a 300 mesh carbon-coated copper grid (Electron Microscopy Sciences). For SEM analysis, $10 \mu \mathrm{L}$ of dispersion was evaporated onto glassy carbon or silicon substrates. Laser diffraction experiments used $10 \mu \mathrm{L}$ of dispersion placed on glass microscope coverslips.

\section{Characterization}

TEM micrographs were acquired on a Phillips EM280 microscope with a $4.5 \AA$ point-topoint resolution operated with an $80 \mathrm{kV}$ accelerating voltage.

HRSEM imaging was performed on a LEO 1530 SEM equipped with a GEMINI field emission column with a thermal field emitter. 
Laser diffraction experiments were carried out using $632.8 \mathrm{~nm}$ (red) helium-neon laser (Melles Griot). A focused beam was projected through a nanocrystal film deposited onto a glass microscope coverslip. The resulting diffraction pattern was projected onto a thin paper screen and a digital camera was used to record images from the far side of the screen.

\section{Contact Angle Measurements}

Substrates were prepared by evaporating a thin layer of gold onto a silicon wafer and immersing the wafer into a Freon solution containing either PFPE-SH or FOMA-SH (1\% by volume) for 18 hours. After removal, the substrates were gently washed with pure Freon to remove unbound thiol and allowed to dry in air. Contact angles for Freon $\left(\theta_{\mathrm{F}}\right)$ and water $\left(\theta_{\mathrm{W}}\right)$ on PFPE and FOMA were measured using a Ramé-hart Inc. goniometer.

$\theta_{\mathrm{W}}$ on the PFPE-coated substrate was $95^{\circ}$, consistent with previous measurements; ${ }^{2}$ repeating the measurements using FOMA-coated substrates gave a lower contact angle $\left(\theta_{\mathrm{W}}=89^{\circ}\right)$, illustrating the higher wettability of FOMA compared with PFPE. The Freon contact angle $\left(\theta_{\mathrm{F}}\right)$ for both treated surfaces was $13^{\circ}$.

A modified version of Young's equation can be used to calculate $\theta_{\mathrm{FW}}$ from the surface tension of water $\left(\gamma_{\mathrm{W}}\right)$ and Freon $\left(\gamma_{\mathrm{F}}\right): \theta_{F W}=\cos ^{-1}\left[\left(\gamma_{W} \cos \left(\theta_{W}\right)-\gamma_{F} \cos \left(\theta_{F}\right)\right) / \gamma_{F W}\right]$. For FOMA-SH, $\theta_{\mathrm{FW}}=130^{\circ}$ and for PFPE-SH $\theta_{\mathrm{FW}}=159^{\circ}$. The Freon-water interfacial tension $\left(\gamma_{\mathrm{FW}}\right)$ was previously measured by us using a pendent drop tensiometer. ${ }^{2}$ The energy required to remove a nanocrystal

from the Freon-water interface into the Freon phase is given by $E=\pi R^{2} \gamma_{F W}\left(1-\left|\cos \theta_{F W}\right|\right)^{2}$, where $R$ is the nanocrystal radius. For $3.2 \mathrm{~nm}$ diameter nanocrystals coated with FOMA-SH, $E \approx 7 k T$. For similar-sized PFPE-SH coated nanocrystals, $E$ would be only approximately $0.2 k T$. 


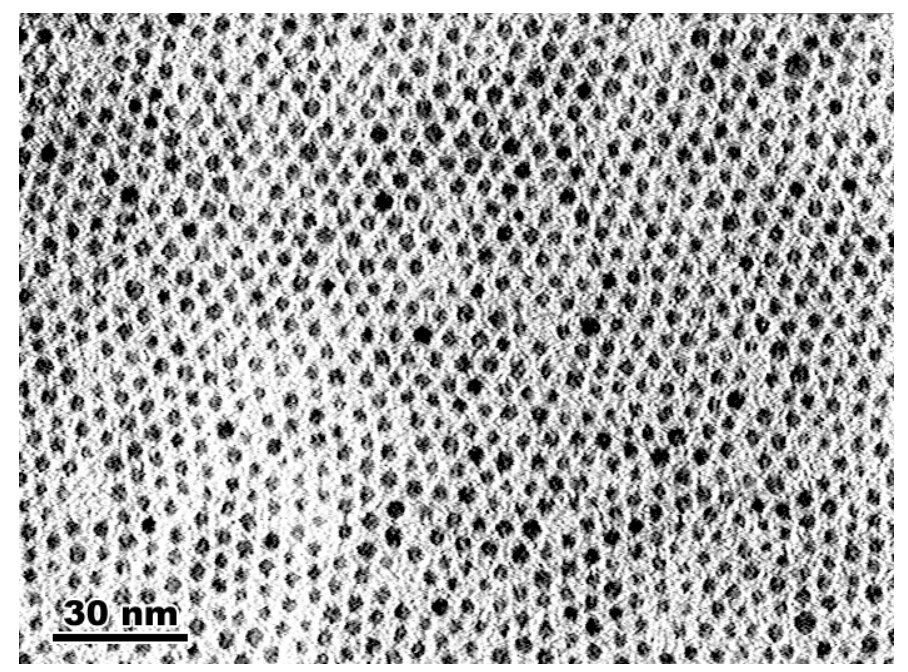

Figure S1: Monodisperse $3.2 \mathrm{~nm}$ (core diameter) FOMA-passivated gold nanocrystals.

\section{References}

1. Shah, P. S.; Novick, B. J.; Hwang, H. S.; Lim, K. T.; Carbonell, R. G.; Johnston, K. P.; Korgel, B. A. Nano Letters 2003, 3, 1671.

2. Shah, P. S.; Sigman, M. B., Jr.; Stowell, C. A.; Lim, K. T.; Johnston, K. P.; Korgel, B. A. Adv. Mater. 2003, 15, 971. 